\title{
Low diagnostic yield of transduodenal endoscopic ultrasound-guided fine needle biopsy using the 19-gauge Flex needle: A large multicenter prospective study
}

\author{
Fabia Attili ${ }^{1}$, Carlo Fabbri' ${ }^{2}$, Ichiro Yasuda 3 , Lorenzo Fuccio ${ }^{4}$, Laurent Palazzo ${ }^{5}$, Ilaria Tarantino ${ }^{6}$, \\ John Dewitt ${ }^{7}$, Leonardo Frazzoni ${ }^{4}$, Mihai Rimbaş, ${ }^{1,8,9}$, Alberto Larghi ${ }^{1}$ \\ ${ }^{1}$ Digestive Endoscopy Unit, Catholic University, Rome, ${ }^{2}$ Unit of Gastroenterology and Digestive Endoscopy, AUSL Bologna \\ Bellaria-Maggiore Hospital, Bologna, ${ }^{4}$ Department of Medical and Surgical Sciences, S. Orsola-Malpighi Hospital, University \\ of Bologna, Bologna, ${ }^{6}$ Department of Diagnostic and Therapeutic Services, Endoscopy Service, IRCCS-ISMETT, Palermo, \\ Italy; ${ }^{3}$ Department of Gastroenterology, Teikyo University Mizonokuchi Hospital, Kawasaki, Japan; ${ }^{5}$ Department of \\ Endoscopy, Trocadero Clinic, Paris, France; ${ }^{7}$ Department of Gastroenterology, Indiana University Health Medical Center, \\ Indianapolis, Indiana, USA; ${ }^{8}$ Department of Gastroenterology, Colentina Clinical Hospital, ${ }^{9}$ Department of Internal \\ Medicine, Carol Davila University of Medicine, Bucharest, Romania
}

\begin{abstract}
Background and Objectives: Previous limited experiences have reported the 19-gauge flexible needle to be highly effective in performing endoscopic ultrasound-guided fine needle biopsy (EUS-FNB) for transduodenal lesions. We designed a large multicenter prospective study with the aim at evaluating the performance of this newly developed needle. Patients and Methods: Consecutive patients with solid lesions who needed to undergo EUS sampling from the duodenum were enrolled in 6 tertiary care referral centers. Puncture of the lesion was performed with the 19-gauge flexible needle (Expect ${ }^{\mathrm{TM}}$ and Slimline Expect ${ }^{\mathrm{TM}} 19$ Flex). The feasibility, procurement yield, and diagnostic accuracy were evaluated. Results: Totally, 246 patients (144 males, mean age $65.1 \pm 12.7$ years) with solid lesions ( 203 cases) or enlarged lymph nodes (43 cases) were enrolled, with a mean size of $32.6 \pm 12.2 \mathrm{~mm}$. The procedure was technically feasible in 228 patients, with an overall procurement yield of $76.8 \%$. Two centers had suboptimal procurement yields (66.7\% and 64.2\%). Major complications occurred in six cases: two of bleeding, two of mild acute pancreatitis, one perforation requiring surgery, and one duodenal hematoma. Considering malignant versus nonmalignant disease, the sensitivity, specificity, positive/negative likelihood ratios, and diagnostic accuracy were $70.7 \%$ ( $95 \%$ confidence interval [CI]: 64.3-76.6), 100\% (95\% CI: 79.6-100), 35.3 (95\% CI: $2.3-549.8) / 0.3$ (95\% CI: $0.2-0.4)$, and $73.6 \%(95 \%$ CI: 67.6-79). On multivariate analysis, the only determinant of successful EUS-FNB was the center in which the procedure was performed. Conclusions: Our results suggest that the use of the 19-gauge flexible needle cannot be widely advocated and its implementation should receive local validation after careful evaluation of both the technical success rates and diagnostic yield.
\end{abstract}

Key words: 19-gauge flexible needle, nitinol endoscopic ultrasound-guided fine needle aspiration needle, transduodenal endoscopic ultrasound-guided tissue acquisition

\begin{tabular}{|l|l|}
\hline \multicolumn{2}{|c|}{ Access this article online } \\
\hline Quick Response Code: & Website: \\
\hline
\end{tabular}

This is an open access article distributed under the terms of the Creative Commons Attribution-NonCommercial-ShareAlike 3.0 License, which allows others to remix, tweak, and build upon the work non-commercially, as long as the author is credited and the new creations are licensed under the identical terms.

For reprints contact: reprints@medknow.com

How to cite this article: Attili F, Fabbri C, Yasuda I, Fuccio L, Palazzo L, Tarantino I, et al. Low diagnostic yield of transduodenal endoscopic ultrasound-guided fine needle biopsy using the 19-gauge Flex needle: A large multicenter prospective study. Endosc Ultrasound 2017;6:402-8.

\section{Address for correspondence}

Dr. Alberto Larghi, Università Cattolica del Sacro Cuore, Largo A. Gemelli 8, Rome 00168, Italy. E-mail: alberto.larghi@yahoo.it Received: 2017-01-16; Accepted: 2017-07-03 
Attili, et al.: EUS-guided 19G flex for transduodenal FNB

\section{INTRODUCTION}

Endoscopic ultrasound-guided fine needle aspiration (EUS-FNA) has become the procedure of choice to obtain samples to reach the definitive diagnosis of lesions of the gastrointestinal (GI) tract and of adjacent organs. ${ }^{[1]}$ EUS-FNA is safe and very accurate, especially when rapid on-site evaluation (ROSE) of the adequacy of the collected specimens is performed. ${ }^{[2-5]}$ However, ROSE is not widely available mostly because the workload consists of surgical pathology and not cytopathology. Moreover, cytology does require a high degree of expertise rarely found outside high volume tertiary care centers. ${ }^{[6]}$ These drawbacks have created a barrier to the dissemination of EUS in the community and in many countries, because the lack of cytology expertise may result in a low diagnostic accuracy and therefore, in a limited perceived utility of EUS. ${ }^{[7]}$

In the last decade, to overcome these limitations of EUS-FNA, various techniques and specifically designed needles to gather tissue core biopsy samples have been developed. ${ }^{[8]}$ In particular, a new technique called EUS-fine needle tissue acquisition (EUS-FNTA) using standard 22-and 19-gauge needles has been developed and evaluated in few studies ${ }^{[9-11]}$ and new histological needles, the Procore ${ }^{\mathrm{TM}}$ needles (Cook Medical, Winston-Salem, North Carolina, USA), have become available and tested in clinical practice. ${ }^{[12-19]} \mathrm{A}$ tissue core biopsy with preserved architecture is critical to diagnose and fully characterize certain neoplasms, such as lymphomas and GI stromal tumors. Moreover, in centers where ROSE is not available, it has been suggested that the performance of EUS-guided fine needle biopsy (EUS-FNB) can result in a greater chance to reach a diagnosis and to provide more tissue for ancillary testing than a typical EUS-FNA sample. ${ }^{[20]}$ Finally, there is increasing interest in evaluating core tissue samples for molecular markers that may serve as prognostic predictors and targets for focused chemotherapy in patients with cancer. ${ }^{[1,22]}$

In a recent review, ${ }^{[23]}$ an algorithm for EUS-tissue acquisition (EUS-TA) of solid lesions depending on the availability of ROSE has been proposed, with the aim of optimizing resource utilization. In institutions with no availability of ROSE, it has been suggested to carry out three EUS-FNB passes for histopathological analysis using 19-gauge needles or any available core biopsy needle. In addition, for lesions accessed from the duodenum, which represent the most difficult sampling position because of the stiffness induced by the needle assembly on the echoendoscope shaft, the authors recommended the use of a 19-gauge needle made of nitinol with increased flexibility. ${ }^{[23]}$ This recommendation was based on a previous study by Varadarajulu et al., ${ }^{[24]}$ who reported the needle to be able to sample transduodenal lesions in all 32 patients included in the evaluation, with a specimen that was procured and diagnostic in all of them.

To test the validity of this recommendation, we performed a prospective multicenter study aimed at evaluating the technical feasibility, procurement yield, and diagnostic accuracy of this newly developed 19 -gauge nitinol flexible needle in patients with solid lesions or enlarged lymph nodes that could be punctured only from the duodenum.

\section{PATIENTS AND METHODS}

\section{Study design}

This was a multicenter prospective study involving 6 centers located in Europe (Italy: Rome, Bologna and Palermo; France: Paris), North America (USA: Indianapolis), and Asia (Japan: Gifu).

The study was approved by the Institutional Review Board of the coordinating center (Catholic University, Rome) and thereafter, by the Ethics Committee of each participating center. The trial has been registered in a publicly accessible registry (ClinicalTrials. gov identifier: NCT02307253).

\section{Study population}

Between June 2013 and March 2015, all consecutive patients with solid lesions who needed to undergo EUS for tissue sampling that had to be performed through the duodenum, such as for deep head/uncinate pancreatic masses, periduodenal lymph nodes, duodenal subepithelial lesions, aortocaval nodes, hilar tumors, right-sided liver lesions, right kidney lesions, right adrenal gland lesions, or periduodenal abdominal masses, were enrolled in the present study.

\section{Selection criteria}

All adults ( $>18$ years of age) referred for EUS-FNA of solid lesions adjacent to or located in the wall of the duodenum with no previous tissue diagnosis were considered eligible. In the presence of a cystic component, the solid part of the lesion should have 
Attili, et al.: EUS-guided 19G flex for transduodenal FNB

been more than $75 \%$ of the total. Patients with uncorrectable coagulopathy as defined by abnormal prothrombin time or partial thromboplastin time that did not normalize after administration of fresh frozen plasma, with altered anatomy of the upper GI tract due to surgery of the esophagus, stomach, and duodenum and those unable to understand and/or read the consent form were excluded from the current study.

\section{Endoscopic ultrasound sampling procedure}

All EUS procedures were performed by advanced endoscopists with the patient under conscious or deep sedation using a conventional linear EUS scope (GF-UC140T, GF-UC180T, Olympus Medical System Europe, USA, and Japan; EG3870UTK, Pentax Europe GmbH, Hamburg, Germany). Once the lesion adjacent to the duodenum was identified by EUS, an eligible puncture site without intervening vessels was selected. Puncture of the lesion using the 19-gauge flexible needle (Expect ${ }^{\mathrm{TM}} 19$ Flex and Slimline Expect $^{\mathrm{TM}} 19$ Flex, Boston Scientific Corp., Marlborough, MA, USA) was performed using the EUS-FNTA technique previously described. ${ }^{[1]}$ Briefly, the needle was prepared before insertion in the channel of the echoendoscope by removing the stylet and attaching to its proximal end the $20-\mathrm{mL}$ syringe already preloaded with $20 \mathrm{~mL}$ of negative pressure. The needle was then advanced only a few millimeters inside the target lesion just under the EUS probe. After opening the lock of the syringe to apply negative pressure, three to four back and forth motions inside the lesion were made using the fanning technique, if possible, to sample different parts of the lesion, ${ }^{[25]}$ together accounting for one needle pass. The lock of the syringe was finally closed, and the needle removed. The collected specimens were immediately placed in formalin for histological examination.

Three needle passes were performed in all patients, and all the collected material was placed in the same collecting vial. In case no material was visible in the container after these three needle passes, the investigator could perform two additional needle passes that were placed in a different collecting vial and these two containers were evaluated separately.

\section{Outcome measurements}

Feasibility was defined as the capability of performing EUS-FNTA through the duodenum by placing the target lesion in the proper position with the insertion of the needle into the lesion. The procurement yield was defined as the percentage of patients in whom a histologically interpretable specimen could be retrieved by EUS-FNTA. A histological adequate sample was defined as an architecturally intact piece of a tissue sample from the targeted lesion that is deemed sufficient for histological evaluation by the histopathologist. The diagnostic accuracy was defined by the rate of correct diagnosis obtained through analysis of the tissue samples acquired with EUS-FNTA. When the histological examination was diagnostic for malignancy, this was considered to be the definitive diagnosis. For patients with EUS-FNTA nondiagnostic for malignancy and/or for a specific benign disease, the presence or exclusion of malignancy was based on following criteria: the histopathological examination of the surgically resected specimen, the results of other diagnostic investigation such as computed tomography-guided and/or laparoscopic biopsy indicating the presence of malignancy, and/or the long-term clinical follow-up, including follow-up imaging. For this purpose, these patients were evaluated for a minimum of 6 months.

\section{Statistical analysis}

Data are presented as proportions and mean \pm standard deviation. Sensitivity, specificity, positive likelihood ratio, negative likelihood ratio, and diagnostic accuracy were computed. The number needed to misdiagnose, defined as the number of patients to be tested in order for one to be misdiagnosed by the test, was computed as $1 /$ (1-accuracy). For the purpose of these analyses, definitive diagnoses were divided into malignant and nonmalignant ones. Inadequate samples for histological evaluation or technical failures were considered as false negative cases. Due to the lack of feasibility and performance data on the use of the Expect ${ }^{\mathrm{TM}}$ Flex 19-gauge needle, no estimation of the sample size has been carried out.

Multivariate logistic regression analysis, adjusted for age, gender, and study center was performed to assess variables independently associated with effective tissue sampling.

Statistical analysis was performed using Stata software version 13 (Stata Statistical Software: Release 13, Stata Corp., College Station, TX, USA).

\section{RESULTS}

The study population

A total of 246 patients (144 males, mean age $65.1 \pm 12.7$ years) with solid lesions (203 cases, 82.5\%) 
Attili, et al.: EUS-guided 19G flex for transduodenal FNB

or enlarged lymph nodes (43 cases, 17.5\%) were enrolled. The mean size of the target lesion was $32.6 \pm 12.2 \mathrm{~mm}$. Among patients with solid lesions, $158(64.2 \%)$ were located in the pancreatic head, $27(11 \%)$ in the uncinate process, and $18(7.3 \%)$ in the bile duct or the right liver lobe. Baseline characteristics are detailed in Table 1.

\section{Endoscopic ultrasound-fine needle tissue acquisition procedure}

The overall performances of the 19 flexible needle are shown in Table 2. EUS-FNTA was done from the duodenal bulb in 150 (61\%) patients and from the second or third duodenal portion in the remaining $96(39 \%)$ cases. The procedure was technically feasible in $228(92.7 \%)$ patients. In 18 patients, the procedure failed because the needle could not be pushed outside the working channel in five cases and to the inability to place the lesion in the proper position to perform the biopsy in the remaining 13 cases $(8$ pancreatic head lesions, 4 uncinate process lesions, and 1 aortocaval lymph node).

\begin{tabular}{|c|c|}
\hline Characteristics & Overall \\
\hline Male sex, $n(\%)$ & $144(58.5)$ \\
\hline Age, years $($ mean $\pm S D)$ & $65.1 \pm 12.7$ \\
\hline \multicolumn{2}{|l|}{ Lesion type, $n(\%)$} \\
\hline Solid mass & $203(82.5)$ \\
\hline Lymph node & $43(17.5)$ \\
\hline \multicolumn{2}{|l|}{ Site of solid lesion, $n(\%)$} \\
\hline Pancreatic head & $158(64.1)$ \\
\hline Uncinate process & $27(11)$ \\
\hline Common bile duct and liver & $18(7.3)$ \\
\hline \multicolumn{2}{|l|}{ Site of lymph node, $n(\%)$} \\
\hline Periduodenal & $12(4.9)$ \\
\hline Aortocaval & $13(5.3)$ \\
\hline Para-right adrenal gland & $1(0.4)$ \\
\hline Hepatic hilum & $8(3.3)$ \\
\hline Retroperitoneal & $9(3.7)$ \\
\hline Maximum diameter, $\mathrm{mm}($ mean $\pm \mathrm{SD})$ & $32.6 \pm 12.2$ \\
\hline Presence of infiltration of other organs, $n(\%)$ & $79(32.1)$ \\
\hline Presence of vascular infiltration, $n(\%)$ & $101(41.1)$ \\
\hline \multicolumn{2}{|l|}{ Center, $n(\%)$} \\
\hline A & $67(27.2)$ \\
\hline B & $45(18.3)$ \\
\hline C & $40(16.3)$ \\
\hline $\mathrm{D}$ & $39(15.8)$ \\
\hline$E$ & $30(12.2)$ \\
\hline $\mathrm{F}$ & $25(10.2)$ \\
\hline
\end{tabular}

SD: Standard deviation
At histological examination, 189 samples were found to be adequate with an overall procurement yield of $76.8 \%$. Technical success rates and procurement yields were stratified by center [Table 3] and ranged from $66.7 \%$ to $97.8 \%$ for the technical success and from $66.7 \%$ to $88 \%$ for the procurement yield, respectively. Two centers had a suboptimal procurement yield of $66.7 \%$ and $64.2 \%$.

Based on the EUS-FNTA histological diagnosis, malignancy was diagnosed in $157(63.8 \%)$ patients, a nonmalignant or a benign disease in $32(13 \%)$ patients, while in $57(23.2 \%)$ cases the sample was judged inadequate (12 cases, $4.9 \%$ ) or without any tissue retrieved (45 cases, 18.3\%). Eight out of 32 cases with a diagnosis of benign disease at EUS-FNTA were a false negative. Considering malignant $v$ s. nonmalignant diseases, the sensitivity, specificity, positive likelihood ratio, negative likelihood ratio, and diagnostic accuracy were 70.7\% (95\% confidence interval [CI]: 64.3-76.6), 100\% (95\% CI: 79.6-100),

Table 2. Performance of the endoscopic ultrasound-guided fine needle biopsy carried out using the 19-gauge flexible needle $(n=246)$

\begin{tabular}{|c|c|}
\hline Flexible 19-gauge performance & Overall \\
\hline \multicolumn{2}{|l|}{ Site of FNA execution, $n(\%)$} \\
\hline Duodenal bulb & $150(61)$ \\
\hline Second and third duodenal portion & $96(39)$ \\
\hline Technical success (FNA feasibility), $n$ (\%) & $228(92.7)$ \\
\hline $\begin{array}{l}\text { Procurement yield (possibility to } \\
\text { obtain histological sample), } n(\%)\end{array}$ & $189(76.8)$ \\
\hline $\begin{array}{l}\text { Possibility to perform } \\
\text { immunohistochemical staining, } n(\%)\end{array}$ & $141(57.3)$ \\
\hline \multicolumn{2}{|l|}{ Diagnosis type from histological sample, $n(\%)$} \\
\hline Malignant lesion & $157(63.8)$ \\
\hline Benign lesion & $32(13)$ \\
\hline No sample & $45(18.3)$ \\
\hline Inadequate sample & $12(4.9)$ \\
\hline \multicolumn{2}{|l|}{$\begin{array}{l}\text { Diagnosis type according to gold } \\
\text { standard diagnostic test, } n(\%)\end{array}$} \\
\hline Malignant lesion & $211(85.8)$ \\
\hline Benign lesion & $35(14.2)$ \\
\hline \multicolumn{2}{|l|}{$\begin{array}{l}\text { Diagnostic performance for } \\
\text { malignant lesions, \% }(95 \% \mathrm{Cl})\end{array}$} \\
\hline Overall accuracy & $73.6(67.6-79)$ \\
\hline Sensitivity & $70.7(64.3-76.6)$ \\
\hline Specificity & $100(79.6-100)$ \\
\hline Positive predictive value & $100(96.5-100)$ \\
\hline Negative predictive value & $27(18.1-37.4)$ \\
\hline Positive likelihood ratio & $35.3(2.3-549.8)$ \\
\hline Negative likelihood ratio & $0.3(0.2-0.4)$ \\
\hline NNM & $3.8(3.1-4.7)$ \\
\hline
\end{tabular}

FNA: Fine needle aspiration, NNM: Number needed to misdiagnose, $\mathrm{Cl}$ : Confidence interval 
Attili, et al.: EUS-guided 19G flex for transduodenal FNB

35.3 (95\% CI: 2.3-549.8), 0.3 (95\% CI: 0.2-0.4), and 73.6\% (95\% CI: 67.6-79), respectively [Table 2]. The number needed to misdiagnose, defined as the number of patients to be tested in order for one not to be diagnosed correctly, was 3.8 (95\% CI: 3.1-4.7).

Major complications related to the EUS-FNTA occurred in six patients $(2.4 \%)$. There were two $(0.8 \%)$ cases of bleeding requiring endoscopic intervention and blood transfusion; $2(0.8 \%)$ cases of mild acute pancreatitis requiring a brief hospitalization; one $(0.4 \%)$ case of duodenal perforation that required surgery; and $1(0.4 \%)$ case of duodenal hematoma that was observed without any intervention.

The yield was also analyzed in terms of probability of success (i.e., obtaining histological sample) with the multivariable binomial logistic regression analysis adjusted for age, sex, center, lesion type, EUS-FNTA site, and lesion site. According to this analysis, the only determinant of successful EUS-FNTA was the center in which the procedure was performed $(P<0.05)$,

Table 3. Technical success rates and yields stratified by center

\begin{tabular}{lcc}
\hline Study center & Technical success rates & Procurement yield \\
\hline A & $64 / 67(95.5)$ & $43 / 67(64.2)$ \\
B & $44 / 45(97.8)$ & $38 / 45(84.4)$ \\
C & $39 / 40(97.5)$ & $34 / 40(85)$ \\
D & $37 / 39(94.9)$ & $32 / 39(82.1)$ \\
E & $20 / 30(66.7)$ & $20 / 30(66.7)$ \\
F & $24 / 25(96)$ & $22 / 25(88)$ \\
\hline
\end{tabular}

Table 4. Multivariate analysis of factors influencing the procurement yield

\begin{tabular}{lcc}
\hline Study variable & OR $(95 \% \mathrm{Cl})$ & $P$ \\
\hline Center (center A as reference) & & \\
B & $2.9(1.02-8.25)$ & 0.046 \\
C & $6.76(1.59-28.79)$ & 0.010 \\
D & $4.12(1.34-12.61)$ & 0.013 \\
E & $1.19(0.42-3.4)$ & 0.743 \\
F & $3.54(1.16-10.84)$ & 0.026 \\
Male sex & $1.1(0.58-2.1)$ & 0.775 \\
Age & $1.01(0.98-1.04)$ & 0.595 \\
Lesion type (lymph node vs. mass) & $1.18(0.31-4.57)$ & 0.811 \\
Lesion diameter & $1.02(0.99-1.06)$ & 0.117 \\
FNA site (second or third duodenal & $1.25(0.6-2.61)$ & 0.550 \\
portion vs. duodenal bulb) & & \\
Lesion site (pancreatic & & \\
head as reference) & & \\
Liver and biliary tree & $0.96(0.21-4.51)$ & 0.960 \\
Intra- and retroperitoneal lymph node & $0.47(0.11-1.99)$ & 0.301 \\
Uncinate process & $0.6(0.05-4.82)$ & 0.523 \\
\hline OR: Odds ratio, Cl: Confidence interval, FNA: Fine needle aspiration &
\end{tabular}

while all the other characteristics did not significantly influence the primary outcome measure [Table 4].

\section{DISCUSSION}

We performed a multicenter prospective study to explore the feasibility, yield, and accuracy of obtaining diagnostic core tissue samples for histological examination using a newly available 19-gauge needle made of nitinol to increase flexibility in a large cohort of consecutive patients with solid lesions or enlarged lymph nodes, which could be approached only from the duodenum. The procedure was technically successful in $92.7 \%$ of the patients, but procurement of a sample for histological examination could be possible in only $76.8 \%$ of the cases, with an overall diagnostic accuracy of $73.6 \%$.

In the last decade, there has been an expansion of techniques and needles specifically designed to gather samples for histological examination under EUS-guidance. ${ }^{[8]}$ The term EUS-guided tissue acquisition has become more and more used to refer to both EUS-FNA and EUS-FNB. ${ }^{[2]}$ This change in EUS practice has been driven by multiple factors: (i) the high dependency of EUS accuracy on ROSE, which is not available in many centers; (ii) the high degree of expertise required to become a skilled cytologist, which has limited the diffusion of EUS outside high volume tertiary care centers; and (iii) the growing interest and need for core tissue specimens to perform molecular profiling to guide targeted therapies for individualized treatment of patients with cancer of the GI tract. ${ }^{[2]]}$

The use of standard 19-gauge needles to perform EUS-FNB has been introduced in 2005 and 2006 by two Japanese investigators in patients with solid pancreatic masses ${ }^{[28]}$ and with mediastinal and/or intra-abdominal lymphadenopathy of unknown origin. ${ }^{[29]}$ They reported an overall diagnostic accuracy of 68.8\% and $98 \%$, respectively. This discrepancy was due to the high failure rate in the first study when sampling was performed transduodenally for pancreatic head and uncinate process masses (5 out of 8 patients, $62.5 \%$ ). ${ }^{[28]}$ Subsequently, Larghi et al. ${ }^{[11]}$ in 2011, published their experience in performing EUS-FNB using a standard 19-gauge needle in 120 patients, in whom a histological sample was deemed more useful than a cytologic one to reach a definitive diagnosis. To increase needle flexibility, they removed the stylet before insertion of the needle in the working channel of the EUS scope. Overall, the procedure was technically successful in all 
but one patient without any complications, with a yield of $96.7 \%$ and a diagnostic accuracy of $93.2 \%$. One drawback of the study was that most of the patients underwent transgastric or transesophageal sampling, without assessing the performance of their technique when done from the duodenum.

To overcome the limitation while using 19-gauge needles for transduodenal sampling, a needle made of nitinol was recently developed with the aim of increasing needle flexibility. Varadarajulu et al., ${ }^{[24]}$ reported this needle to be able to sample transduodenal lesions in all 32 patients evaluated, with specimens procured in all cases for both onsite cytopathological and histological evaluations that resulted diagnostic. Similar results were obtained by Itoi et al., ${ }^{[30]}$ in a bench simulator study comparing the performances of the different 19-gauge needles available, concluding that the resistance to advancing the needle was least with the flexible 19-gauge needle in all experiments, and suggesting that the 19-gauge Flex needle may be the most appropriate choice for puncture in difficult situations, such as the transduodenal approach; however, the authors acknowledge that the results of their study might not hold true for all case scenarios. Apart from these, a study focusing on the interventional capabilities of the 19-gauge flexible needle, though without a comparator, demonstrated equivalent safety, technical and clinical success in the straight versus the angulated endoscope position patient cohorts. ${ }^{[31]}$ In fact, based on these results of the study by Varadarajulu et al. mentioned above, ${ }^{[24]}$ and a subsequent study by Bang et al., ${ }^{[32]}$ an algorithm has been developed in which the flex needle was recommended for sampling lesions through the duodenum when a tissue core biopsy specimen is required, especially in institutions with no availability of ROSE. ${ }^{[23]}$

The findings of our study, with a procurement yield and diagnostic accuracy of only $76.8 \%$ and $73.6 \%$, respectively, redefine the role of the 19-gauge flexible needle for transduodenal EUS-FNB. In our study, the correct diagnosis was missed in about one in every four patients. Since the prevalence of malignant disease in our population was $86 \%$, this finding cannot be considered negligible and should be seriously taken into account. In 2013, Bang et al. ${ }^{[32]}$ proposed an algorithm with the objective of improving technical outcomes of EUS-FNA and suggested to puncture all lesions that need a transduodenal approach by a standard 25 -gauge needle. This study indirectly confirmed that the 19-gauge flexible needle cannot reliably be considered an alternative to thinner needles, due to the disappointing results obtained. This is even more true taking into account the high rate $(2.4 \%)$ of serious complications observed in our cohort.

The results of our study are of particular interest since we showed that the diagnostic performance of the 19-gauge flexible needle has a wide intercenter variability [Table 3], not depending on the expertise of the endoscopist. Indeed, all procedures were performed by endoscopists with a very high expertise, all working in high volume centers for years. One center presented a high rate of technical failures $(33.3 \%)$, while two centers had a diagnostic yield of about $65 \%$. Of note, in one of these centers, despite more than $95 \%$ of the procedures were technically successful, tissue sampling was not judged adequate to formulate a diagnosis in about $30 \%$ of the cases. The intercenter variability was further strenghtened by the multivariable analysis that showed that this variable was the only factor that significantly influenced the diagnostic yield.

The main reason for technical failure was the inability to place the scope in the proper position to puncture the lesion. These failures occurred despite the fact that FNTA technique with the removal of the stylet that should add further flexibility to the needle was used. All centres had a previous experience with the 19-gauge flexible needle before beginning the study enrollment, therefore the suboptimal performance cannot be ascribed to the lack of experience with this type of needle. It is possible that the lack of deformability of the needle may counteract the movement of the distal part of the scope when maximal deflexion is required to place the lesion in the proper position to perform the biopsy.

The main limitation of our study was that only tertiary referral centres with high expertise in EUS-TA were included, thus reducing the external validity. However, the heterogeneity of the results across centres, with variability observed in both technical success rates and diagnostic yield strengthen the reliability of our findings.

\section{CONCLUSIONS}

Our results suggest that the use of the 19-gauge flexible needle for transduodenal FNB cannot be widely suggested and its implementation should receive a local validation, with careful evaluation of both the local technical success rates and diagnostic yield. 
Attili, et al.: EUS-guided 19G flex for transduodenal FNB

\section{Financial support and sponsorship}

The study has been partially supported by a research grant from Boston Scientific Corp.

\section{Conflicts of interest}

Alberto Larghi is a consultant for Boston Scientific Corp. Laurent Palazzo has received educational funds from Boston Scientific Corp..

\section{REFERENCES}

1. Dumonceau JM, Polkowski M, Larghi A, et al. Indications, results, and clinical impact of endoscopic ultrasound (EUS)-guided sampling in gastroenterology: European Society of Gastrointestinal Endoscopy (ESGE) Clinical Guideline. Endoscopy 2011;43:897-912.

2. Eloubeidi MA, Tamhane A, Jhala N, et al. Agreement between rapid onsite and final cytologic interpretations of EUS-guided FNA specimens: Implications for the endosonographer and patient management. Am J Gastroenterol 2006;101:2841-7.

3. Iglesias-Garcia J, Dominguez-Munoz JE, Abdulkader I, et al. Influence of on-site cytopathology evaluation on the diagnostic accuracy of endoscopic ultrasound-guided fine needle aspiration (EUS-FNA) of solid pancreatic masses. Am J Gastroenterol 2011;106:1705-10.

4. Hébert-Magee S, Bae S, Varadarajulu S, et al. The presence of a cytopathologist increases the diagnostic accuracy of endoscopic ultrasound-guided fine needle aspiration cytology for pancreatic adenocarcinoma: A meta-analysis. Cytopathology 2013;24:159-71.

5. Matynia AP, Schmidt RL, Barraza G, et al. Impact of rapid on-site evaluation on the adequacy of endoscopic-ultrasound guided fine-needle aspiration of solid pancreatic lesions: A systematic review and meta-analysis. J Gastroenterol Hepatol 2014;29:697-705.

6. Jhala NC, Jhala DN, Chhieng DC, et al. Endoscopic ultrasound-guided fine-needle aspiration. A cytopathologist's perspective. Am J Clin Pathol 2003;120:351-67.

7. Kalaitzakis E, Panos M, Sadik R, et al. Clinicians' attitudes towards endoscopic ultrasound: A survey of four European countries. Scand J Gastroenterol 2009;44:100-7.

8. Panic N, Larghi A. Techniques for endoscopic ultrasound-guided fine-needle biopsy. Gastrointest Endosc Clin N Am 2014;24:83-107.

9. Larghi A, Noffsinger A, Dye CE, et al. EUS-guided fine needle tissue acquisition by using high negative pressure suction for the evaluation of solid masses: A pilot study. Gastrointest Endosc 2005;62:768-74.

10. Gerke H, Rizk MK, Vanderheyden AD, et al. Randomized study comparing endoscopic ultrasound-guided Trucut biopsy and fine needle aspiration with high suction. Cytopathology 2010;21:44-51.

11. Larghi A, Verna EC, Ricci R, et al. EUS-guided fine-needle tissue acquisition by using a 19-gauge needle in a selected patient population: A prospective study. Gastrointest Endosc 2011;74:504-10.

12. Larghi A, Capurso G, Carnuccio A, et al. Ki-67 grading of nonfunctioning pancreatic neuroendocrine tumors on histologic samples obtained by EUS-guided fine-needle tissue acquisition: A prospective study. Gastrointest Endosc 2012;76:570-7.

13. Iglesias-Garcia J, Poley JW, Larghi A, et al. Feasibility and yield of a new EUS histology needle: Results from a multicenter, pooled, cohort study. Gastrointest Endosc 2011;73:1189-96.
14. Larghi A, Iglesias-Garcia J, Poley JW, et al. Feasibility and yield of a novel 22-gauge histology EUS needle in patients with pancreatic masses: A multicenter prospective cohort study. Surg Endosc 2013;27:3733-8.

15. Bang JY, Hebert-Magee S, Trevino J, et al. Randomized trial comparing the 22-gauge aspiration and 22-gauge biopsy needles for EUS-guided sampling of solid pancreatic mass lesions. Gastrointest Endosc 2012;76:321-7.

16. Vanbiervliet G, Napoléon B, Saint Paul MC, et al. Core needle versus standard needle for endoscopic ultrasound-guided biopsy of solid pancreatic masses: A randomized crossover study. Endoscopy 2014;46:1063-70.

17. Lee YN, Moon JH, Kim HK, et al. Core biopsy needle versus standard aspiration needle for endoscopic ultrasound-guided sampling of solid pancreatic masses: A randomized parallel-group study. Endoscopy 2014;46:1056-62.

18. Iwashita T, Nakai $Y$, Samarasena JB, et al. High single-pass diagnostic yield of a new 25-gauge core biopsy needle for EUS-guided FNA biopsy in solid pancreatic lesions. Gastrointest Endosc 2013;77:909-15.

19. Attili F, Petrone G, Abdulkader I, et al. Accuracy and inter-observer agreement of the Procore ${ }^{\mathrm{TM}} 25$ gauge needle for endoscopic ultrasound-guided tissue core biopsy. Dig Liver Dis 2015;47:943-9.

20. Varadarajulu S, Hawes RH. The changing paradigm in EUS-guided tissue acquisition. Gastrointest Endosc Clin N Am 2014;24:1-7.

21. Braat H, Bruno M, Kuipers EJ, et al. Pancreatic cancer: Promise for personalised medicine? Cancer Lett 2012;318:1-8.

22. Brais RJ, Davies SE, O'Donovan M, et al. Direct histological processing of EUS biopsies enables rapid molecular biomarker analysis for interventional pancreatic cancer trials. Pancreatology 2012;12:8-15.

23. Varadarajulu S, Hasan MK, Bang JY, et al. Endoscopic ultrasound-guided tissue acquisition. Dig Endosc 2014;26 Suppl 1:62-9.

24. Varadarajulu S, Bang JY, Hebert-Magee S. Assessment of the technical performance of the flexible 19-gauge EUS-FNA needle. Gastrointest Endosc 2012;76:336-43.

25. Bang JY, Magee SH, Ramesh J, et al. Randomized trial comparing fanning with standard technique for endoscopic ultrasound-guided fine-needle aspiration of solid pancreatic mass lesions. Endoscopy 2013;45:445-50.

26. Wani S, Muthusamy VR, Komanduri S. EUS-guided tissue acquisition: An evidence-based approach (with videos). Gastrointest Endosc 2014;80:939-59.e7.

27. Larghi A, Eguia V, Hassan C, et al. Economic crisis: The right time to widen endoscopic ultrasound utilization. Endoscopy 2014;46:80-1.

28. Itoi T, Itokawa $\mathrm{F}$, Sofuni $\mathrm{A}$, et al. Puncture of solid pancreatic tumors guided by endoscopic ultrasonography: A pilot study series comparing trucut and 19-gauge and 22-gauge aspiration needles. Endoscopy 2005;37:362-6.

29. Yasuda I, Tsurumi H, Omar S, et al. Endoscopic ultrasound-guided fine-needle aspiration biopsy for lymphadenopathy of unknown origin. Endoscopy 2006;38:919-24.

30. Itoi T, Itokawa F, Sofuni A, et al. Evaluation of 19-gauge endoscopic ultrasonography aspiration needles using various echoendoscopes. Endosc Int Open 2013;1:24-30.

31. Kumbhari V, Peñas I, Tieu AH, et al. Interventional EUS using a flexible 19-gauge needle: An international multicenter experience in 162 patients. Dig Dis Sci 2016;61:3552-9.

32. Bang JY, Ramesh J, Trevino J, et al. Objective assessment of an algorithmic approach to EUS-guided FNA and interventions. Gastrointest Endosc 2013;77:739-44. 